\title{
Assistência de Enfermagem ao paciente portador de esquizofrenia
}

\author{
Nursing assistance for patients with schizophrenia \\ Asistencia de enfermería para pacientes con esquizofrenia
}

Diêgo Wilton Ricardo dos Reis

ORCID: https://orcid.org/0000-0002-8072-2139 Faculdade Asa de Brumadinho, Brasil

E-mail: diegowrreis@gmail.com

Raniere Vitória Quares Nascimento ORCID: https://orcid.org/0000-0001-6251-6086

Faculdade Asa de Brumadinho, Brasil E-mail: ranierevitoria@outlook.com

Tiago de Oliveira Porto

ORCID: https://orcid.org/0000-0002-3152-4910

Faculdade Asa de Brumadinho, Brasil

E-mail: toporto25@gmail.com

Vanessa Moura da Costa Campos

ORCID: https://orcid.org/0000-0002-4229-7717

Faculdade Asa de Brumadinho, Brasil

E-mail: vanessa.moura46@yahoo.com

Sarah Cristina de Oliveira

ORCID: https://orcid.org/0000-0002-0849-0919 Faculdade Asa de Brumadinho, Brasil E-mail: saracristina1521@gmail.com

Tatiane Barros Lacerda

ORCID: https://orcid.org/0000-0003-2403-6051 Faculdade Asa de Brumadinho, Brasil E-mail: tatiane-barros@hotmail.com

Bruna Taiane Campos Nunes

ORCID: https://orcid.org/0000-0002-8982-6532 Faculdade Asa de Brumadinho, Brasil

E-mail: brunataianecampos@gmail.com

Graciete Mendes de Souza

ORCID: https://orcid.org/0000-0001-6095-3645

Faculdade Asa de Brumadinho, Brasil E-mail: gracietmendes@gmail.com

Milena Sandi Pereira

ORCID: https://orcid.org/0000-0003-0543-9496 Faculdade Asa de Brumadinho, Brasil E-mail: milenasandip@gmail.com

Rhayana Monise Paiva Silva

ORCID: https://orcid.org/0000-0003-3987-6897 Faculdade Asa de Brumadinho, Brasil E-mail: paivarhayana4@gmail.com

\begin{abstract}
Resumo
Introdução: A esquizofrenia, atualmente, é considerada como um dos principais problemas de saúde, caracterizada por mudanças do pensamento, memória, percepção e emoção, provocando grave perturbação mental. Objetivos: Descrever a atuação da equipe de enfermagem na assistência ao paciente com esquizofrenia. Método: Trata-se de uma revisão integrativa da literatura, cuja seleção ocorreu no período entre agosto de 2020 e abril de 2021. Resultados: No manejo de pacientes psíquicos, o papel da enfermagem dentro da equipe multidisciplinar, deve refletir sobre a prática e ampliação da sua visão profissional, abandonando as atividades rotineiras do modelo manicomial e clínico, buscando maior interação e aproximação com os sujeitos. As práticas assistenciais incluem o auxílio às demandas apresentadas pela família, cuidado à pessoa com transtorno mental e avaliação da sobrecarga familiar. Além do fornecimento de informações sobre a doença, bem como estimular a perseverança e adesão ao tratamento, apoio por meio da escuta, auxílio nos momentos de crise, incentivo à família durante o processo de reabilitação, e lidar com os efeitos colaterais do tratamento no aspecto biológico. Conclusões: Em virtude dos fatos apresentados, é evidente que os profissionais de enfermagem necessitam de ações como capacitação e educação permanente, renovando o conhecimento, discutindo as relações profissional-usuário, desenvolvendo o trabalho em equipe e a criação de espaços de cuidado. A enfermagem frente aos pacientes com esquizofrenia deve considerar algumas habilidades, tais como: a comunicação efetiva, o respeito, a empatia, e a capacidade de não julgar o outro.
\end{abstract}


Palavras-chave: Esquizofrenia; Saúde mental; Enfermagem psiquiátrica; Transtornos mentais; Cuidados de enfermagem; Papel do profissional de enfermagem.

\begin{abstract}
Introduction: Schizophrenia is currently considered one of the main health problems, characterized by changes in thinking, memory, perception and emotion, causing severe mental disorder. Objectives: To describe the role of the nursing team in assisting patients with schizophrenia. Method: This is an integrative literature review, whose selection took place between August 2020 and April 2021. RESULTS: In the management of psychic patients, the role of nursing within the multidisciplinary team, should reflect on the practice and expansion from his professional vision, abandoning the routine activities of the asylum and clinical model, seeking greater interaction and approximation with the subjects. Care practices include assisting the demands presented by the family, caring for people with mental disorders and assessing family burden. In addition to providing information about the disease, as well as encouraging perseverance and adherence to treatment, support through listening, assistance in times of crisis, encouragement to the family during the rehabilitation process, and dealing with the side effects of treatment in terms of biological. Conclusions: Due to the facts presented, it is evident that nursing professionals need actions such as training and continuing education, renewing knowledge, discussing professional-user relationships, developing teamwork and creating spaces for care. Nursing in the face of patients with schizophrenia must consider some skills, such as: effective communication, respect, empathy, and the ability to not judge others.

Keywords: Schizophrenia; Mental health; Psychiatric nursing; Mental disorders; Nursing care; Nurse's role; Mental health services.

\section{Resumen}

Introduccíon: La esquizofrenia se considera en la actualidad uno de los principales problemas de salud, caracterizado por cambios en el pensamiento, la memoria, la percepción y la emoción, provocando un trastorno mental severo. Objetivos: Describir el papel del equipo de enfermería en la asistencia a pacientes con esquizofrenia. Método: Se trata de una revisión integradora de la literatura, cuya selección se realizó entre agosto de 2020 y abril de 2021. Resultados: En el manejo de pacientes psíquicos, el rol de la enfermería dentro del equipo multidisciplinario, debe reflexionar sobre la práctica y expansión desde su visión profesional, abandonando las actividades rutinarias del asilo y modelo clínico, buscando una mayor interacción y aproximación con los sujetos. Las prácticas de cuidado incluyen la atención a las demandas presentadas por la familia, el cuidado de las personas con trastornos mentales y la evaluación de la carga familiar. Además de brindar información sobre la enfermedad, así como fomentar la perseverancia y adherencia al tratamiento, el apoyo a través de la escucha, la ayuda en tiempos de crisis, el estímulo a la familia durante el proceso de rehabilitación y el manejo de los efectos secundarios del tratamiento en el aspecto biológico. Conclusiones: A la vista de los hechos presentados, es evidente que los profesionales de enfermería necesitan acciones como la formación y educación continua, la renovación de conocimientos, la discusión de las relaciones profesionalusuario, el desarrollo del trabajo en equipo y la creación de espacios de atención. Los pacientes de enfermería con esquizofrenia deben considerar algunas habilidades, tales como: comunicación efectiva, respeto, empatía y la capacidad de no juzgar al otro.
\end{abstract}

Palabras clave: Esquizofrenia; Enfermería psiquiátrica; Rol del profesional de enfermería.

\title{
1. Introdução
}

Dados do Ministério da Saúde afirmam que cerca de 3\% da população brasileira manifesta algum transtorno mental grave ou prevalente, além de $12 \%$ carecer de algum atendimento frequente ou esporádico em unidade de saúde mental. (Czarnobay, J. 2015)

De acordo com D' Assunção, Dos Santos, Lino e Silveira (2016), Casaleiro, Seabra e Caldeira (2017), Giacon e Galera, (2006 apud Humerez, 2012), a esquizofrenia, um dos principais problemas de saúde da atualidade, é caracterizada por mudanças do pensamento, memória, percepção e emoção, que provoca uma grave perturbação mental e se apresenta prevalente em aproximadamente quatro indivíduos por cada mil habitantes ao longo da vida, manifestando-se entre o final da adolescência e por volta dos 30 anos, ocasionando grande sofrimento para o doente e familiares. Uma das consequências desse transtorno é o suicídio, que contempla cerca de 5 a $6 \%$ dos indivíduos diagnosticados. Em consonância com Ferreira et al (2015), a esquizofrenia é o transtorno mental mais discriminado socialmente, devido a apresentar sintomas que no geral são associados a "loucura", como anormalidade psicomotora e alucinações. 
A incidência de transtornos mentais tem aumentado gradativamente no mundo nos últimos anos. Estima-se que as doenças crônicas e os distúrbios mentais representam 59\% do total de número de óbitos em todo o planeta e, aproximadamente, 650 milhões de pessoas apresentam algum tipo de transtorno mental, sendo considerado quatro dos 10 principais motivos de incapacitação no mundo. (Castro, Furegato e Santos, 2019)

Pessoas que têm relação de parentesco em primeiro grau com esquizofrenia possuem dez vezes mais chances de desenvolver a doença, se comparado com quem não tem casos na família. Em casos de gravidez gemelar, a conciliação é maior em gêmeos monozigóticos do que em relação aos gêmeos dizigóticos, porém os fatores ambientais também são importantes para o desenvolvimento do transtorno. Existe ainda a relação entre a exposição do feto à infecção viral, de acordo com as observações, onde se apresenta mais intensa no período do segundo trimestre de gestação, pois é nesse período que é considerado crítico, onde ocorre a formação do sistema límbico e cortical (Cardoso, Carvalho \& Matos, 2020).

Apesar da complexidade e importância para a área da saúde, a maioria dos países dedicam somente $2 \%$ dos recursos à saúde mental (Ferraz et al., 2019; Santos, 2018). Contudo, segundo o Ministério da Saúde [Brasil, M.S] (2020), a assistência a pessoas com necessidades de tratamento em saúde mental tem sido tratada com prioridade. No ano de 2019 , cerca de R $\$ 97$ milhões de reais foram investidos na Rede de Atenção Psicossocial (RAPS), representando um aumento de quase $200 \%$ se comparado ao ano de 2018. Com estes investimentos foram disponibilizados novos serviços, como a construção de 92 Centros de Atenção Psicossocial (CAPS), 63 Serviços de Residência Terapêutica (SRT), 181 leitos de saúde mental em hospitais, 5 Unidades de Acolhimento (AU) e 29 equipes multiprofissionais.

Segundo Ferraz et al. (2019), para que o atendimento aos pacientes esquizofrênicos atenda todas as suas necessidades, se faz necessário a presença de uma equipe multiprofissional. A enfermagem desempenha papel de extrema importância no que tange aos cuidados ofertados aos pacientes, onde é de sua competência contribuir com a qualidade de vida destes e seus familiares.

De acordo com Silva et al. (2018) e Silva et al. (2020), a assistência de enfermagem aos pacientes esquizofrênicos deve basear-se em teorias fundamentadas nos princípios da Reforma Psiquiátrica Brasileira e da Política Nacional de Humanização, considerando as necessidades e direitos dos pacientes enquanto seres humanos, para que alcance qualidade e forneça o cuidado humanizado. Conforme afirmam Castro et al. (2019), Oliveira, Júnior e Furegato (2019), a prevalência dos acolhimentos dos pacientes em sofrimento psíquico é realizado pelo profissional de enfermagem. Deste modo, os cuidados ofertados aos pacientes devem promover a assistência humanizada e estabelecer o vínculo enfermeiro-paciente, proporcionando o acolhimento efetivo.

Diante dos dados encontrados, essa pesquisa tem como objetivo descrever a atuação da equipe de enfermagem na assistência ao paciente com esquizofrenia.

\section{Metodologia}

Trata-se de uma revisão integrativa da literatura, que utiliza a pesquisa bibliográfica com a finalidade de identificar o papel da equipe de enfermagem na assistência ao paciente com esquizofrenia. Segundo Gil (2002, p.44), “[...] a pesquisa bibliográfica é desenvolvida com base em material já elaborado, constituído principalmente de livros e artigos científicos". Deste modo, o presente artigo embasou-se em livros, artigos, portarias e diretrizes nacionais que abordassem o tema principal.

A seleção dos artigos foi realizada no período entre agosto de 2020 e abril de 2021 e os descritores utilizados foram: esquizofrenia, saúde mental, enfermagem psiquiátrica, enfermagem, transtornos mentais, cuidados de enfermagem, papel do profissional de enfermagem e serviços de saúde mental. Os artigos foram selecionados por meio da plataforma BIREME combinando dois descritores como o operador booleano and, utilizando os seguintes critérios de inclusão: publicações dos 
últimos 05 anos, disponíveis em português e coleção brasileira. Ao final da pesquisa, foram encontrados um total de 354 artigos que, após leitura prévia dos títulos e resumos, foram selecionadas 70 publicações, conforme demonstra a tabela abaixo.

A Tabela 1 demonstra o número de artigos encontrados e artigos selecionados após aplicação dos critérios de inclusão estabelecidos acima.

Tabela 1. Seleção de artigos para produção científica do estudo.

\begin{tabular}{ccc}
\hline Combinação de descritores & Artigos encontrados & Artigos selecionados \\
\hline Esquizofrenia [and] Enfermagem & 17 & 6 \\
Esquizofrenia [and] Fisiopatologia & 5 & 3 \\
Esquizofrenia [and] Enfermagem & 12 & 2 \\
Psiquiátrica & 5 & 5 \\
Esquizofrenia [and] Cuidados de & & \\
Enfermagem & 20 & 4 \\
Papel Do Prof. De Enfermagem & & \\
[and] Serviços De Saúde Mental & 10 & 47 \\
Papel Do Prof. De Enfermagem & & \\
[and] Transtornos mentais & 285 & \\
Enfermagem Psiquiátrica [and] & & \\
Saúde Mental & &
\end{tabular}

Fonte: Plataforma BIREME, 2021. Tabela elaborada pelos acadêmicos.

\section{Resultados e Discussão}

Após leitura e análise do conteúdo desses artigos, os repetidos e àqueles que não estavam em consonância com a temática da pesquisa foram descartados. Também foram excluídos os arquivos caracterizados como tese, monografia ou trabalho de conclusão de curso. Desta forma, após leitura rigorosa dos artigos, foram selecionadas 19 publicações para a elaboração da pesquisa.

\subsection{Aspectos históricos da psiquiatria}

Segundo De Carvalho (2012), no período colonial brasileiro, raro são os achados sobre portadores de doenças mental no país. Até o século XVIII, os "loucos", assim denominados, que viviam sem moradia, eram recolhidos e mantidos acondicionados em porões construídos nos fundos das casas, totalmente excluídos da sociedade, onde eram submetidos a condições sub-humanas, tais como castigos físicos e contenções inapropriadas, como forma de punição pelo comportamento não apropriado assim julgado por eles.

As primeiras unidades da Santa Casa de Misericórdia, locais que eram destinados para os doentes e abrigo para os desvalidos, tinham como objetivo ser um projeto social. Em 1543, foi inaugurada a primeira Santa Casa de Misericórdia em Santos, posteriormente outras cidades seguiram o exemplo e criaram suas próprias instituições (De Carvalho, 2012).

No final do século XIX houve um aumento súbito de leitos psiquiátricos no Brasil e, em meados do mesmo século, foram criados no país os primeiros locais destinados à assistência ao doente mental (De Carvalho, 2012).

Após muitas queixas de maus tratos advindos da população que viviam nessas unidades da Santa Casa de Misericórdia e por pedido de alguns médicos higienistas, foi discutida a necessidade da criação de um hospital destinado a este 
fim. Através do decreto no 82/1841, do imperador Dom Pedro II, em 1852, cria-se então o Hospício Pedro II na cidade do Rio de Janeiro, oficialmente a primeira instituição psiquiátrica brasileira como descreve Miranda-Sá e Medeiros (2006 como citado em De Carvalho, 2012, p. 15). Em seguida, outros diversos centros psiquiátricos foram construídos em todo o território nacional, como afirmam Kirschbaum e Bastos (1997, 2007 como citado em De Carvalho, 2012, p. 15).

De acordo com Menezes da Silva e da Silva Jr (2006 como citado em De Carvalho, 2012, p 16) em 1903 foi criado um decreto $\mathrm{n}^{\circ}$ 1.132/1903 que legalizou a reclusão e concedeu amplos poderes aos psiquiatras, cuja atuação restringiu somente a fiscalização da saúde mental.

No período republicano, o Estado e a psiquiatria brasileira enfrentou crises, abandono e mudanças em suas administrações. Diante de tais mudanças, acontecimentos e devido à falta de profissionais, criou se no Brasil a Escola Profissional de Enfermeiros e Enfermeiras, que seguiu o exemplo da escola já existente em Salpêtrière, na França segundo Melo e Carvalho (1986;1976 como citado em De Carvalho, 2012, p.16). Contudo até o século XX, o profissional da enfermagem tinha sua função apenas como mediador da organização local e não como profissional de intervenções terapêuticas (De Carvalho, 2012).

Cordeiro et al. (2019) afirma que, até o final da década de 1970, o modelo de assistência à saúde mental era de um atendimento resumido em isolamento, exclusão, grandes internações e condutas impróprias das equipes de saúde, sendo conhecido como a época do "corredor da loucura", onde o atendimento não tinha nenhuma assistência adequada, humanizada e não oferecia o mínimo de cidadania aos doentes mentais.

Diante destes fatos, surgiu a necessidade da reforma psiquiátrica e leis que autenticavam esse processo até a atualidade. Somente no final do século XX, surgiram as ideias e ações sobre atendimento psiquiátrico extra-hospitalar, ou seja, os hospitais-dia, os CAPS, e os Núcleos de Atenção Psicossocial (NAPS) considerando-se a primeira experiência concreta de desconstrução manicomial no Brasil (De Carvalho, 2012; Hirdes, 2009 como citado em De Carvalho, 2012, p. 17).

O aumento pela procura de atendimento à saúde mental nas Unidades Básicas de Saúde (UBS) originou dificuldades, devido ao despreparo das equipes de saúde no acolhimento desses pacientes, já que era prematura a busca por este atendimento nessas unidades, tornando-o desorganizado, despreparado e com uma defasagem de critérios para uma assistência específica. Além dos profissionais apresentarem certo receio ao atendê-los, devido à falta de conhecimento das patologias e do preconceito existente. (Cordeiro et al., 2019)

\subsection{A esquizofrenia: conceitos e fisiopatologia}

A esquizofrenia é uma doença mental que atinge a zona "central do eu" e altera toda a estrutura do indivíduo, sua percepção da realidade e sua relação com o mundo. O indivíduo portador de esquizofrenia, por não ter o entendimento "normal" da realidade, representa o estereótipo de "louco", não possuindo juízo crítico ou razão sobre si e sua condição, perdendo a liberdade de escapar de suas próprias produções mentais. (Humerez, 2012)

A esquizofrenia é uma psicopatologia crônica caracterizada por mudanças persistentes, como delírios, alucinações, distúrbios da fala, alterações no comportamento, inquietação e irritabilidade, retardo emocional, reações violentas e isolamento social. (Cardoso et al., 2020)

Em consonância com Cunha, Lembi, Martins, Nicolato e Silva (2015), a fisiopatologia da esquizofrenia não é totalmente compreendida. Os estudos têm mostrado que a inflamação e o estresse oxidativo são resultados do desequilíbrio entre a formação de radicais livres que podem danificar o DNA, membrana celular e matriz extracelular, e a habilidade dos sistemas antioxidantes de neutralizá-los, que exercem um papel essencial na fisiopatologia de vários transtornos mentais, como especificamente a esquizofrenia. 
Dalgalarrondo (2008), afirma que no primeiro episódio deste transtorno, a intervenção é de suma importância para o início do tratamento, porém, a demora da procura para iniciar a terapia pode influenciar na piora do prognóstico.

Segundo a Classificação Estatística Internacional de Doenças e Problemas Relacionados com a Saúde (CID-10), a esquizofrenia pode ser classificada em 9 tipos de transtornos, sendo eles: Esquizofrenia, Esquizofrenia paranoide, Esquizofrenia hebefrênica, Esquizofrenia catatônica, Esquizofrenia indiferenciada, Depressão pós-esquizofrênica, Esquizofrenia residual, Esquizofrenia simples e Esquizofrenia não especificada Associação Americana De Psiquiatria [APA] (2014).

Os sintomas causados por essa doença podem ser agrupados em dois tipos: positivos e negativos, porém essa ideia não está relacionada ao sentido qualitativo de bom ou ruim. Nos sintomas positivos se encaixam as alucinações, delírios, agitação psicomotora, dentre outros. Já os sintomas negativos são geralmente os déficits, que implicam na pobreza de conteúdo do pensamento, fala e a incapacidade de sentir emoções e prazer. Para diagnosticar o paciente com esse transtorno é necessário atentar-se a outros sintomas como a alucinação auditiva áudio-verbal (vozes externas ou audição do próprio pensamento), e o pensamento persecutório (sensação de ter ações controladas). (Humerez, 2012)

Os sinais e sintomas indicativos devem persistir por pelo menos seis meses, vivenciados por no mínimo um mês da fase ativa, contendo dois ou mais destes: alucinações, discursos, comportamentos incoerentes e delírios. (D' Assunção et al., 2016)

\subsection{Incidência e prevalência da Esquizofrenia}

Os índices de incidência e prevalência da Esquizofrenia tem aumentado gradativamente no mundo nos últimos anos. A Tabela 2 ilustra os dados epidemiológicos da esquizofrenia conforme os autores utilizados na confecção do artigo.

Tabela 2. Dados epidemiológicos sobre a Esquizofrenia.

Autor (es)

Assunção et al. (2016), Casaleiro et al. (2017), Giacon; Galera (2006) apud Humerez (2012, p. 71)

Assunção et al. (2016), Casaleiro et al. (2017), Giacon; Galera (2006) apud Humerez (2012, p. 71)

Castro et al. (2019) e Czarnobay, J. (2015)

Castro et al. (2019) e Czarnobay J. (2015)

Czarnobay J. (2015)

Czarnobay J. (2015)

Maria da Guia Clementino Ferraz et al., (2019) e Santos (2018)

Brasil, M.S (2020)

Dados epidemiológicos

Afeta 4 indivíduos a cada 1.000 habitantes

Geralmente a doença manifesta se no final da adolescência e por volta dos 30 anos

Doenças crônicas e os distúrbios mentais representam 59\% do total de número de óbitos por todo o planeta

650 milhões de pessoas apresentam algum tipo de transtorno mental

3\% da população brasileira manifesta algum transtorno mental grave ou prevalente

$12 \%$ da população brasileira carece de algum atendimento frequente ou esporádico em unidade de saúde mental

A maioria dos países dedicam somente $2 \%$ dos recursos à saúde mental

Cerca de R $\$ 97$ milhões de reais foram investidos na RAPS em 2019

Fonte: Assunção et al. (2016); Casaleiro et al. (2017); Giacon e Galera (2006) apud Humerez (2012) p.71; Castro et al. (2019); Czarnobay J. (2015); Maria da Guia Clementino Ferraz et al., (2019); Santos (2018); Brasil, M.S (2020). Tabela elaborada pelos acadêmicos.

É evidente nesses dados que, milhões de pessoas no mundo possuem algum tipo de transtorno mental, entre eles a esquizofrenia. Contudo, as doenças crônicas e os distúrbios mentais representam 59\% das mortes em todo o planeta, dados estes preocupantes uma vez que, a sociedade e os profissionais de saúde menosprezam essa especialidade. 
Está explícito que a maioria dos países dedicam somente 2\% dos recursos a este ramo. Em contrapartida, o Ministério da Saúde alega que a saúde mental tem sido tratada como uma prioridade neste governo, e que houve um aumento significativo em relação ao ano de 2018.

\subsection{Assistência de enfermagem na esquizofrenia}

Ferraz et al. (2019) informa que, as modificações realizadas no decorrer dos anos na atenção à Saúde Mental vivenciam uma reorganização dos serviços de saúde, estabelecendo-se uma substituição das redes, propondo mudanças nos cuidados realizados aos pacientes com sofrimento psíquico, promovendo a sua reinserção na sociedade, através dos níveis de atenção e complexidade.

$\mathrm{O}$ atendimento à pessoa com sofrimento psicossocial é uma das atribuições dos serviços públicos de saúde, independentemente de serem especializados em saúde mental, como os centros de atenção psicossocial (CAPS) e as unidades básicas de saúde (UBSs). Sobre a estrutura da atenção psicossocial, a construção de um novo lugar social para os portadores de transtornos mentais e a transformação das práticas institucionalizantes às quais os mesmos, em sua maioria, eram submetidos sob a égide do modelo hospitalocêntrico, hegemônico no país até o início dos anos 2000. (Rosa et al., 2021)

Em consonância com Santos (2018) e Ferraz et al. (2019), a atuação do enfermeiro na rede de atenção psicossocial é ampla, podendo ser exercida em: atenção básica em saúde, atenção psicossocial especializada, urgência e emergência, atenção residencial de caráter transitório, atenção hospitalar e estratégias de desinstitucionalização, além da reabilitação psicossocial. Possibilitando a discussão coletiva de casos e tomadas de decisões, elaboração de projetos de caráter interdisciplinar, bem como a adoção de ações transversais entre as equipes de saúde nos diversos níveis da Rede de Atenção à Saúde.

Embora os profissionais de saúde e pesquisadores consideram essencial, a atenção à saúde mental em centros de atenção primária à saúde enfrenta uma série de desafios, incluindo medo e incompetência reportado aos profissionais que estabelecem contato direto com muitas pessoas (tradicionalmente chamadas de "loucas") ligadas à fragilização na formação e capacitação profissional. (Rosa et al., 2021)

Considerando que a esquizofrenia não é sinônimo de violência, os problemas enfrentados pelos pacientes com esquizofrenia em suas vidas diárias e entender como os enfermeiros irão trabalhar com estes pacientes e suas famílias é fundamental. Estes devem mostrar à sociedade que desde que o paciente portador do transtorno seja bem tratado e cuidado, ele pode viver em um ambiente social de forma satisfatória, promovendo uma integração social e da família. (Cardoso, et al., 2020)

Historicamente, o enfermeiro em saúde mental assumia papel de repressão e vigilância, porém, a reforma psiquiátrica transformou a assistência a esses pacientes enfatizando a reinserção social, desenvolvimento da autonomia do sujeito, convivência e a comunicação com o outro, a participação em grupo e o desenvolvimento do pragmatismo. (Ferraz et al., 2019).

A equipe multidisciplinar promove novos moldes do cuidar, com acolhimento em grupo e individuais, oficinas, atividades físicas e lúdicas. Auxiliam no processo de tratamento biopsicossocial e na reinserção social, possibilitando a aproximação aos serviços de atendimento e comunidade, colaborando para uma adesão ao tratamento de sucesso. (D' Assunção et al., 2016).

Ferraz et al., (2019), evidencia que o papel do enfermeiro no manejo de pacientes psíquicos dentro da equipe multidisciplinar precisa refletir sobre a prática e ampliação da sua visão profissional, abandonando as atividades cotidianas do modelo manicomial e clínico, buscando maior aproximação e interação com os sujeitos aos quais destinam seu cuidado através dos novos modelos de assistência. 
Segundo Casaleiro et al. (2017) o fornecimento de serviços técnicos advindos da enfermagem em saúde mental, acompanha o paciente e seus familiares favorecendo o bem-estar da família, minimizando a sobrecarga e promovendo segurança, além de tranquilidade a todos os envolvidos.

Tal qual, Humerez (2012) e D' Assunção et al. (2016) remete como competência do enfermeiro: o auxílio às demandas apresentadas pela família, cuidado à pessoa com transtorno mental e avaliação da sobrecarga familiar. Incube também o fornecimento de informações sobre a doença, bem como estimular a perseverança e adesão ao tratamento, apoio por meio da escuta, auxílio nos momentos de crise, incentivo a família durante o processo de reabilitação, além de lidar com os efeitos colaterais do tratamento no aspecto biológico. Em harmonia, Coelho et al. (2020) declara que a enfermagem deve compor de habilidades que promovam a relação de ajuda profissional com o paciente assistido, como a comunicação efetiva, o respeito, a empatia, e a capacidade de não julgar o outro.

Em contrapartida, diante da rotina sobrecarregada que a equipe de enfermagem enfrenta, Oliveira, Alves, Porto e Cavalcanti (2016, p. 3933) diz que “[...] as enfermeiras resolvem os problemas que são levados ao seu conhecimento [...] pelos auxiliares de enfermagem e também pelos médicos”. Desta forma, muitas das vezes, a enfermagem realiza ações focadas no cuidado físico, como administração de medicamentos, vigilância e observação do comportamento dos pacientes. (Oliveira et al., 2016)

Neste mesmo âmbito, Cardoso et al. (2020) conclui que a assistência de enfermagem somente será assertiva quando os profissionais colocarem em prática o conhecimento científico adquirido, reduzindo as negligências em torno da profissão, devido às características da patologia e a falta de conhecimento específico.

Um estudo desenvolvido por Castro et al. (2019) evidenciou que a enfermagem é responsável pela maioria dos acolhimentos aos pacientes de doença mental, nos serviços especializados destinados a este fim. Portanto, a assistência humanizada e o estabelecimento do vínculo enfermeiro-paciente são fatores que promovem o acolhimento efetivo. (Castro et al., 2019; Oliveira; Júnior; Furegato, 2019)

Segundo Castro et al. (2019) e Oliveira, Junior e Furegato (2019), no contexto da internação psiquiátrica, a enfermagem possui papel essencial na construção do plano terapêutico conforme as necessidades daquele indivíduo.

A Sistematização da Assistência de Enfermagem (SAE) permite planejar o cuidado individualizado à pessoa com adoecimento mental e funciona como ferramenta efetiva e imprescindível na enfermagem psiquiátrica (Humerez, 2012).

Neste olhar, uma pesquisa desenvolvida por Bard, Feijó, Paz e Linch (2020) identificou que, as prevalências dos diagnósticos de enfermagem em saúde mental estão relacionadas aos sentimentos do paciente assistido, especificamente: ansiedade, medo, tristeza, agressividade, estresse, negação e relações familiares. Na mesma pesquisa, foi evidenciado algumas intervenções de enfermagem mais comuns nos serviços de saúde mental, tais como:

[...] dar esclarecimentos sobre tratamento, estar atento aos riscos físicos e emocionais, estimular saída do quarto, orientar sobre higiene do sono, incluir familiares no tratamento, promover e permitir escolhas quando possível, evitar exaustão física e mental do paciente, realizar feedback positivo ao enfrentamento, dar suporte emocional, promover estímulos cognitivos, orientar comportamento adequado, realizar escuta ativa e mediar conflitos interpessoais. (Bard, et al., 2020, p. 1170)

Conforme Silva et al. (2018), a assistência de enfermagem em saúde mental deve se basear nos preceitos da Reforma Psiquiátrica Brasileira e da Política Nacional de Humanização para que alcance qualidade e forneça o cuidado humanizado. De acordo com Silva et al. (2020), nesta perspectiva, a enfermagem deve ser norteada por uma base teórica que respeite todas as necessidades e direitos dos pacientes como seres humanos. 
Maftum, Silva, Borba, Brusamarello e Czarnobay (2017), relata que com a reforma psiquiátrica, o profissional de enfermagem passou a atuar ativamente dentro da equipe multiprofissional, deixando de ser cumpridora de procedimentos técnicos, passando a agir com autonomia profissional, tornando-se ativo na assistência aos portadores de transtornos mentais.

A reforma psiquiátrica colaborou para a evolução da autonomia do paciente, historicamente o enfermeiro na saúde mental assumia somente papel de repressão e vigilância. A partir da criação dos CAPS e NAPS, o atendimento aos usuários com transtornos mentais tornou-se de fácil acesso, possibilitando uma assistência eficaz, humanizada e satisfatória. Essa reestruturação dos serviços de saúde mental propícia a reinserção do indivíduo na comunidade, buscando um novo olhar do cuidado. (Ferraz et al., 2019)

A atuação do enfermeiro pode ser exercida em todos os níveis de atenção no atendimento aos pacientes esquizofrênicos, como afirmam Santos (2018) e Ferraz et al. (2019). Dessa forma, os cuidados de enfermagem devem estar direcionados à busca de interações, promovendo aproximação entre a equipe e o paciente, possibilitando o cuidado humanizado.

Os cuidados de enfermagem além de assumirem caráter assistenciais, tais como, administração de medicamentos, observação comportamental, adesão medicamentosa, dentre outros, deve estar relacionada ao esclarecimento de dúvidas sobre toda a fase terapêutica, riscos emocionais, promoção de autonomia ao paciente, realização de avaliações, as quais possibilitam acompanhamento da evolução, além da escuta dos familiares, como pode ser observado na Tabela 3.

Tabela 3. Principais cuidados de enfermagem aos pacientes com Esquizofrenia.

\begin{tabular}{|c|c|}
\hline Autor (es) & Cuidados \\
\hline Ferraz et al., (2019) & $\begin{array}{l}\text { Aproximação e interação da equipe de enfermagem com os sujeitos por meio dos novos modelos } \\
\text { de assistência. }\end{array}$ \\
\hline Casaleiro et al. (2017) & $\begin{array}{l}\text { Favorecimento do bem-estar da família, minimizando a sobrecarga, promovendo segurança e } \\
\text { tranquilidade. }\end{array}$ \\
\hline $\begin{array}{l}\text { D' Assunção et al., } \\
\text { (2016) e Humerez } \\
\text { (2012) }\end{array}$ & $\begin{array}{c}\text { Auxílio às demandas apresentadas pela família, bem como estimular a perseverança e adesão ao } \\
\text { tratamento, amparo nos momentos de crise, além de lidar com os efeitos colaterais do tratamento } \\
\text { no aspecto biológico. }\end{array}$ \\
\hline Oliveira et al. (2016) & $\begin{array}{l}\text { Realização de ações focadas no cuidado físico, como administração de medicamentos, vigilância } \\
\text { e observação do comportamento dos pacientes. }\end{array}$ \\
\hline Castro et al. (2019) & Acolhimento aos pacientes, além da construção do plano terapêutico. \\
\hline Bard et al. (2020) & $\begin{array}{l}\text { Estar atento aos riscos físicos e emocionais, estimular saída do quarto, orientar sobre higiene do } \\
\text { sono, promover e permitir escolhas quando possível, evitar exaustão física e mental do paciente, } \\
\text { realizar feedback positivo ao enfrentamento, promover estímulos cognitivos, orientar } \\
\text { comportamento adequado, realizar escuta ativa e mediar conflitos interpessoais. }\end{array}$ \\
\hline Coelho et al. (2020) & $\begin{array}{l}\text { Promover relação de ajuda profissional ao paciente assistido, comunicação efetiva, respeito, } \\
\text { empatia e capacidade de não julgar o outro. }\end{array}$ \\
\hline
\end{tabular}

Fonte: Humerez (2012), D' Assunção et al., (2016), Oliveira et al. (2016), Casaleiro et al. (2017), Castro et al. (2019), Ferraz et al, (2019), Bard et al. (2020), Coelho et al. (2020). Tabela elaborada pelos autores.

Conforme os dados demonstrados na tabela acima, a assistência de enfermagem aos pacientes com esquizofrenia, por meio dos novos moldes de cuidado, consiste em: aproximação e interação da equipe de enfermagem com os sujeitos, contribuindo para o bem-estar familiar, diminuindo a exaustão e promovendo segurança e tranquilidade; auxílio às necessidades pontuadas pela família, estimular a adesão ao tratamento, amparo nos momentos de crise; atentando-se aos riscos físicos e emocionais, estimular o convívio social e cuidados básicos de higiene pessoal, promovendo a autonomia quando possível. (Ferraz et al., 2019; Casaleiro et al., 2017; D' Assunção et al., 2016; Humerez, 2012)

Outrossim, acolher e construir um plano terapêutico holístico, minimizar o cansaço psicomotor do paciente, realizar feedback positivo ao enfrentamento, proporcionar melhorias intelectuais, promover escuta ativa e comunicação efetiva, mediar 
confrontos interpessoais, estimular relação de ajuda profissional, além de desempenhar ações concentradas no cuidado físico, como administração de medicamentos, vigilância e observação do comportamento dos pacientes, incluem práticas assistenciais de enfermagem no manejo ao paciente esquizofrênico. (Oliveira et al., 2016; Castro et al., 2019; Bard et al., 2020; Coelho et al., 2020)

As práticas assistenciais devem ser norteadas por materiais científicos, buscando respeitar o paciente esquizofrênico em todas as suas dimensões, embasadas nos conceitos da Reforma Psiquiátrica Brasileira.

\section{Considerações Finais}

Em virtude dos fatos mencionados, essa patologia é julgada pela população como "loucura", entretanto alguns estudos mostram que a inflamação e o estresse oxidativo podem estar associados a esse desequilíbrio. Os dados epidemiológicos demonstram uma estatística assustadora, que reforça ainda mais o quanto os profissionais estão despreocupados e despreparados para promover a plenitude do bem-estar biopsicossocial.

Portanto, a atuação da enfermagem diante do diagnóstico de esquizofrenia deve ocorrer em todos os níveis de atenção, traçando métodos juntamente com a equipe multidisciplinar, para a evolução dos casos e melhoria na adesão do tratamento. Desta forma, possibilita a diminuição da sobrecarga de seus familiares e promove a tranquilidade e segurança de todos os envolvidos.

Levando em consideração esses aspectos, a enfermagem também desempenha funções acolhedoras aos pacientes esquizofrênicos, sendo assim é imprescindível ferramentas afetivas durante todo o cuidado, de modo que proporcione apoio nos momentos de crises, desenvolva um plano terapêutico humanizado a partir da SAE e preserve a individualidade do cliente.

Dado o exposto, é evidente que os profissionais de enfermagem necessitam de ações como capacitação e educação permanente, renovando o conhecimento, discutindo as relações profissional-usuário, desenvolvendo o trabalho em equipe e a criação de espaços de cuidados. Dessa forma, a falta de formação especializada pode ocasionar a replicação de algumas práticas profissionais, que estão contra os princípios da reforma psiquiátrica, fazendo com que a equipe de enfermagem não se sinta segura em desenvolver suas ações.

\section{Referências}

Associação Americana De Psiquiatria. (2014) Manual Diagnóstico e Estatístico de transtornos mentais. (5a ed,)

Bard, N. D., Feijó, I. O., Ipuchima, J. R., Paz, A. A., \& Linch, G. F. da C. (2020). Nursing diagnoses and interventions in mental health used in hospital admissions units: integrative Review / Diagnósticos e intervenções de enfermagem em saúde mental utilizados em unidades de internação hospitalares: revisão integrativa. RPCFO, 12, 1165-1171. https://doi.org/10.9789/2175-5361.rpcfo.v12.8029

Brasil, M.S. (2020) Agência Saúde. Saúde Mental: investimento cresce 200\% em 2019. Brasília.

Cardoso, A. O. de J., de Carvalho, G. T., \& de Matos, T. S. (2020). A prática de enfermagem frente aos pacientes portadores de esquizofrenia. Revista Eletrônica Acervo Enfermagem, 5, e5118. https://doi.org/10.25248/reaenf.e5118.2020

Casaleiro, T., Seabra, P., \& Caldeira, S. (2017) Eficácia das intervenções de enfermagem na sobrecarga da família da pessoa com esquizofrenia: revisão de literatura. CuidArte, Enferm, p. 287-292. Disponível em: http://www.webfipa.net/facfipa/ner/sumarios/cuidarte/2017v2/287.pdf.

Coelho, J., Sampaio, F., Teixeira, S., Parola, V., Sequeira, C., Fortuño, M. L., \& Merino, J. R. (2020). A relação de ajuda como intervenção de enfermagem: Uma scoping review. Revista Portuguesa de Enfermagem de Saúde Mental, (23), 63-72. https://dx.doi.org/10.19131/rpesm.0274

Cordeiro, G. F. T., Ferreira, R. G. S., Almeida Filho, A. J., Santos, T. C. F., Figueiredo, M. A. G., \& Peres, M. A. A. Atendimento em saúde mental na atenção primária à saúde no período pré-reforma psiquiátrica. (2019) Atendimento em saúde mental na atenção primária à saúde no período pré-reforma psiquiátrica, $10.5935 / 1415-2762.20190076$

Cunha, M. G., Lembi, P. J., Martins, L. C. A., Nicolato, R., \& Silva, M. A. R. (2015). Papel do estresse oxidativo na fisiopatologia da esquizofrenia. Rev Med Minas Gerais 25 (Supl 5): S30-S34: 10.5935/2238-3182.20150108

Czarnobay, J. (2015). Adesão ao uso de psicofármacos pelo portador de transtorno mental: percepções do enfermeiro. Curitiba, Universidade Federal do Paraná, 2015. 94 p. https://acervodigital.ufpr.br/handle/1884/41793. 
Research, Society and Development, v. 10, n. 7, e8110716444, 2021

(CC BY 4.0) | ISSN 2525-3409 | DOI: http://dx.doi.org/10.33448/rsd-v10i7.16444

D'assunção, C. F., Santos, A. L. D., Lino, F. A., Silveira, E. A. A. (2016). A enfermagem e o relacionamento com os cuidadores dos portadores de esquizofrenia. Revista De Enfermagem Do Centro Oeste Mineiro, DOI: 10.19175.

Dalgalarrondo, P. (2008). Psicopatologia e Semiologia dos Transtornos Mentais. (2a ed.), Artes Médicas.

De Carvalho, M. B. (2012) Inserção Histórica da Psiquiatria no Brasil. In: De Carvalho, M.B. Psiquiatria para enfermagem. Rideel. Cap. 1, p. 13-29.

De Castro, S. A. D., Furegato, A. R. F., \& Santos, J. L. F. (2019). Egressos de internação psiquiátrica acompanhados na rede de serviços de saúde. Revista de enfermagem e Atenção à Saúde, 7(1). https://doi.org/10.18554/reas.v7i1.2055

Ferraz, M. G. C., De Sousa, M. I. B., De Araújo, A. P., De Sousa, S. C., Benevides, K. G. C. B., \& Silva, K. C. O. (2019). Atuação do enfermeiro no atendimento aos usuários com sofrimento psíquico. Revista de Enfermagem UFPE on line, 13. https://doi.org/10.5205/1981-8963.2019.242131

Ferreira, F. N., Fernandino, D. C., Souza, G. R. M., Ibrahim, T. F., Fukino, A. S. L., Araújo, N. C., \& Vidal, C. E. L. (2015) Avaliação das Atitudes de Estudantes da Área da Saúde em relação a Pacientes Esquizofrênicos. Revista brasileira de educação médica, 39(4), 542-549, https://doi.org/10.1590/1981$52712015 v 39 n 4 \mathrm{e} 01562014$

Humerez, D.C. (2012) Transtornos do pensamento e assistência de enfermagem. Psiquiatria para enfermagem,4, 69-89.

Maftum, A. M., Silva, A. G., Borba, L. O., Brusamarello, T., \& Czarnobay, J. (2017). Mudanças ocorridas na prática profissional na área da saúde mental frente à reforma psiquiátrica brasileira na visão da equipe de enfermagem Changes in professional practice in the mental health area against brazilian psychiatric reform in the vision of the nursing team. RPCFO, 9(2), 309-314. https://doi.org/10.9789/2175-5361.2017.v9i2.309-314

Oliveira, R. M. D., Júnior, A. C. S., \& Furegato, A. R. F. (2019). Cuidados de enfermagem mais valorizados e frequentes durante internação psiquiátrica. Revista de enfermagem e Atenção à Saúde, 7. https://doi.org/10.18554/reas.v7i1.2272.

Oliveira, R. M. P., Alves, M., Porto, I. S., \& Cavalcanti, P. C. S. (2016). A clínica de enfermagem psiquiátrica e suas novas tecnologias de cuidado. Revista de Pesquisa: Cuidado é Fundamental Online, 8, (1), 3922-3934. http://dx.doi.org/10.9789/2175-5361.2016.v8i1.3922-3934

Rosa, D. C. J., Lima, D. M., Miranda, L., \& Peres, R. S. (2021). Paciente problema: imaginário coletivo de enfermeiros acerca do usuário com diagnóstico de esquizofrenia. Revista de saúde coletiva, 31 (310108), 1-21. http://dx.doi.org/10.1590/S0103-73312021310108.

Santos, R. C. A., Junior, J. M. P., \& Miranda, F. A. N. (2018). Rede de atenção psicossocial: adequação dos papéis e funções desempenhados pelos profissionais. Revista Gaúcha de Enfermagem, 39, 1-10. https://doi.org/10.1590/1983-1447.2018.57448

Silva, J., Ribeiro, H., Fernandes, M., \& Rocha, D. (2020). O cuidar de enfermagem em saúde mental na perspectiva da reforma psiquiátrica. Enfermagem em Foco, 11(1). https://doi.org/10.21675/2357-707X.2020.v11.n1.2743

Silva, P., Silva, D., Rodrigues, C., Santos, N., Barbosa, S., Souto, V., \& Gusmão, R. (2018). Cuidado clínico de enfermagem em saúde mental. Revista de Enfermagem UFPE on line, 12(11), 3133-3146. https://doi.org/10.5205/1981-8963-v12i11a236214p3133-3146-2018 